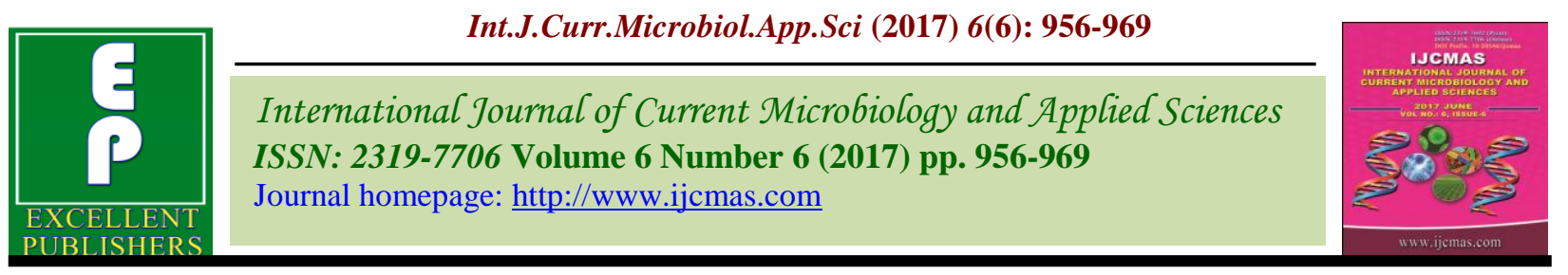

Original Research Article

https://doi.org/10.20546/ijcmas.2017.606.112

\title{
Study of Heterosis and Pollen Fertility in CGMS Based Pigeonpea [Cajanus cajan (L.) Millspaugh] Hybrids
}

\author{
Neetu Soni and P.T. Patel* \\ Seed Spices Research Station, S.D. Agricultural University, Jagudan 382710, District, \\ Mehsana, Gujarat State, India \\ *Corresponding author
}

A B S T R A C T

Thirty CGMS-based pigeonpea [Cajanus cajan (L.) Millspaugh] hybrids were synthesized by crossing six CMS lines with five ' $R$ ' lines and evaluated to study yield potential with the performance of their R-lines. Result indicated that the male sterile lines exhibited 100 Keywords $\quad$ per cent pollen sterility and R- line acts as good restorer and pollen fertility varied from 98 to 100 per cent. All crosses exhibited pollen fertility ranged from 92 to 100 per cent. Most of the hybrids showed standard heterosis in desired direction for yield and its contributing traits with high fertility restoration. The range of standard heterosis over GTH 1 for seed yield per plant was from -27.29 (CMS GT 301 A x GTR 52) to 69.79 per cent (CMS GT 603 A x GTR 52). The best cross combinations for seed yield were CMS GT 603 A X GTR 52 (69.79\%), CMS GT 603 A X GTR 23 (64.59\%), CMS GT 288 A X GTR 95 $(57.22 \%)$ and CMS GT 603 A X GTR 95 (53.76\%), CMS GT 601 A X GTR 95 (49.41 $\%)$, CMS GT 33 A X GTR 18 (47.69\%), CMS GT 603 A X GTR 8 (46.81\%), CMS GT 601 A X GTR $52(37.30 \%)$,CMS GT 302 A X GTR 8(33.39\%) and CMS GT 301 A X GTR 95 (28.61\%). These hybrids had high per se performance for grain yield, more standard heterosis with one or more its contributing traits viz. number of pods per plant, biological yield, leaf area and harvest index and having high pollen fertility status suggested that these hybrids can be directly exploited commercially after evaluating their performance in wide range of environment. These crosses can also be used to throw-off transgressive segregants for the improvement of yield and its attributing traits.

\section{Introduction}

Pigeonpea [Cajanus cajan (L.) Millsp.] Is an environment friendly crop, which is a staple food across the country and plays an important role in national economic and nutritional security with qualities of improving soil fertility and structure? It is an important food legume of semi-arid tropical regions of Asia, Africa and the Caribbean islands, where it is grown on over $5 \mathrm{~m}$ ha (FAO, 2013). Productivity of pigeonpea worldwide in comparisons to cereal is very low and stagnant due to several biotic and abiotic stress and in spite of decades of research and development programs, the mean productivity of the crop could not cross the barrier of $800 \mathrm{~kg} / \mathrm{ha}$. Hybrid breeding technology, developed by ICRISAT to break the yield plateau which is based on cytoplasmic nuclear male sterility (CMS) and natural out-crossing. The first stable CMS line for commercial exploitation could be developed by Tikka et al., (1997), GT-288A 
with its maintainer, GT-288B utilizing as A2 cytoplasm source. Later pollen fertility restorer lines were identified (Chauhan et al., 2004, Acharya et al., 2005). For getting good yield, male fertility restoration of hybrid plant is important. According to Kaul (1988) once a fertility restorer $(\mathrm{R}-)$ line is crossed with male sterile (A-) plant, the dominant fertility restoring nuclear gene produces certain proteins in F1 plants and thus repairs the defective mitochondrial genome of the plant to produce male fertile hybrid plants. In pigeonpea two dominant genes (Rf1 and Rf2) have been identified (Saxena et al., 2011), which impart fertility restoration to the hybrid plants. This cytoplasmic-genic male sterility system contains A line with $\mathrm{S}$ (rr), B line with $\mathrm{F}$ (rr) and $\mathrm{R}$ line with $\mathrm{S} / \mathrm{F}$ (RR) and consequently, first CGMS based hybrid SKNPH-10 (GTH-1) has been released for cultivation in Gujarat (Patel et al., 2004 and Majmudar et al., 2004). In the primary gene pool the frequency of fertility restoring genes is fairly high and so far over 150 good restorers have been identified in different maturity groups (Saxena et al., 2014), heterosis using cms lines and pollen fertility restorer lines for grain yield studied by Patel and Tikka (2014a,b).

The hybrid technology is based on three major components namely, male sterility and its genetic maintenance system, stable fertility restoration, and cost effective mass pollination mechanism. The development of stable CMS systems in pigeonpea is a boon to the breeders and it has provided a platform to enhance the pace of research and development of hybrid pigeonpea. The utilization of CMS system for the development of commercial hybrids in pigeonpea would be possible of improvement of seed yield by developing hybrids or by selecting transgressive segregants from the crosses showing high heterotic response as pollen fertile restorer parent.

\section{Materials and Methods}

The thirty hybrids obtained through hand pollination during kharif 2014 at Main Pulses Research Station, Sardarkrushinagar Dantiwada Agricultural University, SardarKrushinagar using newly developed six cytoplasmic male sterile lines and five diverse restorers as pollinators in a line $\mathrm{x}$ tester mating design (Table 1). The experiments conducted during kharif 2015. The latitude and longitude were $24012^{\prime} \mathrm{N}$ and $72012^{\prime} \mathrm{E}$. The altitude and soil type were $154.5 \mathrm{~m}$ and loamy sand, for these location. The experimental materials comprised of six cytoplasmic male sterile line used as fertile counterpart, five pollen fertility restorer line as male parents, thirty synthesized hybrids and standard check GTH 1 and evaluated using randomized block design with three replications for each location. Each genotype was represented by a single row plot of $4.0 \mathrm{~m}$ length. The inter and intra row distances were 60 and $20 \mathrm{~cm}$, respectively which accommodated 20 plants per plot. All the agronomical practices and plant protection measures were followed for raising the good crop. Observations were recorded on five randomly selected competitive plants of each geno $\neg$ type in each replication for various characters i.e. plant height $(\mathrm{PH})(\mathrm{cm})$, number of branches per plant (BP), number of pods per plant $(\mathrm{PP})$, pod length $(\mathrm{PL})(\mathrm{cm})$, Number of seeds per pod (SP), 100-seed weight (g) (TW), seed yield per plant (g) (SY), biological yield per plant (g) (BY). Days to flower (DF) on the basis of $50 \%$ plants of each genotype flowered, days to maturity (DM) on the basis of $80 \%$ plants of each genotype matured were recorded. The protein content (PC) was estimated in percentage by using Nuclear Magnetic Resonance Technique (Tiwari et al., 1974). Harvest Index was calculated by using following formula [(Economic yield/Biological yield $) \mathrm{x}$ 100]. The replication wise mean values were 
used in statistical analysis. The replication wise mean value of each genotype for various characters was used for statistical genetical analysis. Heterosis was estimated as per cent increase or decrease in the mean value of F1 hybrid over standard check i.e., standard heterosis Meredith and Bridge (1972) for each character. The pollen fertility/sterility observations were recorded on parental lines ('A' lines and male parents) during crossing season and for F1 and check GTH 1 were recorded during evaluating season at the initiation of flowering stage. The fertility status was determined. The test for fertility and sterility of pollen grains was done as per aceto-carmine stain method by Alexander, 1969.

The test comprised staining pollen grains in 2 gm carmine solution was prepared by dissolving in hot $45 \%$ glacial acetic acid, boil for half an hour and cool and filter. Five well developed flower buds were collected randomly from different parts of each plant at the time of anthesis (9-10 AM). From each bud, the anthers were collected on a glass slide and crushed with a drop of $2 \%$ acetocarmine stain and examined under a light microscope. The mean value of pollen fertility/sterility of five plants was considered as pollen fertility/sterility (\%) for that genotype (Saxena et al., 2011).

\section{Results and Discussion}

The analysis of variance for all the characters under study was presented in Table 1. There were significant differences among the parents for all the characters except protein content in parents. This indicated the presence of adequate amount of variability in parents (lines and testers) for most of the characters under studied. Mean sum of squares due to lines were significant for all the characters except seed yield, branches per plant and biological yield. Mean sum of squares due to testers were significant for all the characters except number of pods and pod length. Further, mean sum of squares due to hybrids and parent vs. hybrids were significant for all the characters except for number of seeds per pod, branches per plant and protein content, which indicated the presence of enormous heterosis for these traits. Mean sum of squares due to line vs. tester significant for all characters except 100 seed weight.

\section{Heterosis estimated over check (Standard Heterosis)}

The main aim of plant breeder is to evolve high yielding varieties. The yield is the attribute that receive greatest importance. It is a complex trait associated with number of component traits which are under polygenic control. The perusal of the results revealed (Table 2) that thirteen hybrids exhibited significant and positive standard heterosis. The standard heterosis ranged from -27.29 (CMS GT 301 A x GTR 52) to 69.79 per cent (CMS GT 603 A x GTR 52).

Three most positive heterotic crosses for GTH 1 in descending order were CMS GT 603 A X GTR 52 (69.79\%) and CMS GT 603 A X GTR 23 (64.59\%) and CMS GT 288 X GTR 95 (57.22). Similar findings were also evident by the findings of Patel and Tikka (2008), Acharya et al., (2009), Dheva et al., (2009), Gite et al., (2009), Phad et al., (2009), Sameer Kumar et al., (2009), Sarode et al., (2009), Chadirakala et al., (2010), Shoba and Balan (2010), Gupta et al., (2011) and Lay et al., (2011).

Early flowering and maturity is one of the desirable traits in hybrid pigeonpea as it helps in escaping drought. Majority of hybrids showed late maturity. The estimates of standard heterosis for days to flowering revealed (Table 2) that five hybrids for significant heterosis in desired direction 
(negative) with a range from -13.03 (CMS GT 33 A X GTR 95) to 22.18 per cent CMS GT 301 A X GTR 18) and for days to maturity ranged from ranged from -3.59 (CMS GT 33 A X GTR 23) to 18.90 per cent (CMS GT 601 A X GTR 23). Heterosis in both negative and positive direction for days to flowering had also evident by Kumar and Srivastva (1998), Wankhade et al., (2005), Baskaran and Muthiah (2006), Wanjari et al., (2007), Patel and Tikka (2008), Sarode et al., (2009), Chandirakala et al., (2010) and Vaghela et al., (2011).

The estimates of heterosis for plant height revealed (Table 2) that majority of hybrids exhibit taller stature over standard check. The standard heterosis ranged from-4.47 per cent (CMS GT 33 A X GTR 52) to 41.76 per cent (CMS GT 603 A X GTR 52). Similar findings were also recorded by Rana (1990), Aghav et al., (1997), Chandirakala and Raveendran (2002) and Chandirakala (2010) et al., for plant height.

The standard heterosis for number of branches per plant varied from -21.41 (CMS GT 33 A X GTR 18 and CMS GT 601 A X GTR 52) to 27.31 per cent (CMS GT $301 \mathrm{~A} X$ GTR 95). The hybrids with positive heterosis for number of pods per plant are desirable to increase yield. The standard heterosis ranged from -36.00 (CMS GT 301 A X GTR 52) to 15.97 per cent (CMS GT 301 A X GTR 95). Results were found agreement with Phad et al., (2009) and Shoba and Balan (2010) for number of branches per plant and number of pods per plant.

The results presented in table 2 revealed that out of 30 hybrids, none of the hybrids evinced significant and positive standard heterosis for number of seeds per pod. The range of variation of standard heterosis from -24.21 (CMS GT 301 A x GTR 18) to 1.69 per cent (CMS GT 301 A X GTR 52, CMS GT 302 A X GTR 18, CMS GT 33 A X GTR 95, CMS GT 288 A X GTR 23, CMS GT 603 A X GTR 18, CMS GT 603 A X GTR 23 and CMS GT 603 A X GTR 95). Out of 30 hybrids, 6 hybrids exhibited (Table 2) significant positive standard heterosis for 100seed weight, with a range varying from -4.02 (CMS GT 33 A x GTR 8) to 8.92 per cent (CMS GT 302 A x GTR 8). The results were found agreement with Chandirakala and Raveendran (2002), Yadav and Singh (2004), Aher et al., (2006) and Baskaran and Muthiah (2006) for number of seed per pod and 100seed weight.

For pod length, results revealed (Table 2) that twelve hybrids significant positive standard heterosis. The standard heterosis for this trait ranged from -6.64 (CMS GT 301 A x GTR 18) to 23.67 per cent (CMS GT 288 A x GTR 95). The results were similar with the findings of Thiruvengadam and Muthiaha (2012) and Patel and Tikka (2014).

Table.1 Analysis of variance showing mean sum of squares for different characters in pigeonpea

\begin{tabular}{|l|c|c|c|c|c|c|c|c|}
\hline \multicolumn{1}{|c|}{ Parents } & d.f. & $\begin{array}{c}\text { Days to } \\
\text { flowering }\end{array}$ & $\begin{array}{c}\text { Days to } \\
\text { maturity }\end{array}$ & $\begin{array}{c}\text { Plant height } \\
\mathbf{( c m )}\end{array}$ & $\begin{array}{c}\text { Number } \\
\text { of } \\
\text { branches } \\
\text { per plant }\end{array}$ & $\begin{array}{c}\text { Number of } \\
\text { pods per } \\
\text { plant }\end{array}$ & $\begin{array}{c}\text { Number } \\
\text { of } \\
\text { seeds } \\
\text { per pod }\end{array}$ & $\begin{array}{c}\text { Pod } \\
\text { length } \\
\text { (cm) }\end{array}$ \\
\hline Replication & 2 & 1.00 & 0.58 & 91.57 & 0.48 & 155.05 & 0.015 & 0.10 \\
\hline Parents & 10 & $1903.85^{* *}$ & $422.92^{* *}$ & $1143.53^{* *}$ & $11.80^{* *}$ & $902.35^{* *}$ & $0.38^{* *}$ & $1.22^{* *}$ \\
\hline Lines & 5 & $608.36^{* *}$ & $486.10^{* *}$ & $539.30^{* *}$ & 0.91 & $1647.27^{* *}$ & $0.43^{* *}$ & $1.77^{* *}$ \\
\hline Testers & 4 & $37.56^{* *}$ & $243.76^{* *}$ & $759.46^{* *}$ & $16.15^{* *}$ & 127.42 & $0.39^{* *}$ & 0.03 \\
\hline Lines $\boldsymbol{v s}$ Testers & 1 & $1333.30^{* *}$ & $823.65^{* *}$ & $727.45^{*}$ & $48.89^{* *}$ & $277.34^{* *}$ & $0.09^{* *}$ & $3.26^{*}$ \\
\hline Parents $\boldsymbol{v}$ Hybrids & 1 & $198.78^{* *}$ & $456.87^{* *}$ & $12844.06^{* *}$ & 1.66 & $280331.20^{* *}$ & 0.11 & $2.90^{* *}$ \\
\hline Hybrids & 29 & $329.75^{* *}$ & $258.22^{* *}$ & $727.45^{* *}$ & 3.72 & $4430.83 * *$ & 0.35 & $0.33^{* *}$ \\
\hline Error & 80 & 6.33 & 15.37 & 134.75 & 0.45 & 255.49 & 0.05 & 0.08 \\
\hline
\end{tabular}




\begin{tabular}{|l|c|c|c|c|c|c|c|}
\hline \multicolumn{1}{|c|}{ Parents } & d.f & $\begin{array}{c}\text { 100 seed } \\
\text { weight (g) }\end{array}$ & $\begin{array}{c}\text { Seed yield } \\
\text { per plant (g) }\end{array}$ & $\begin{array}{c}\text { Harvest } \\
\text { index (\%) }\end{array}$ & $\begin{array}{c}\text { Total } \\
\text { protein } \\
\text { content } \\
(\mathbf{\%})\end{array}$ & $\begin{array}{c}\text { Biological } \\
\text { yield }(\mathbf{g})\end{array}$ & $\begin{array}{c}\text { Leaf area } \\
\left(\mathbf{c m}^{\mathbf{2}}\right)\end{array}$ \\
\hline Replication & 2 & 0.29 & 50.92 & 7.55 & 0.08 & 52.55 & $13869.79^{* *}$ \\
\hline Parents & 10 & $0.60^{* *}$ & $195.72^{* *}$ & $33.91^{* *}$ & 0.19 & $1903.85^{* *}$ & $699443.80^{* *}$ \\
\hline Lines & 5 & $0.79^{* *}$ & 67.29 & $28.31^{* *}$ & $0.76^{* *}$ & 878.27 & $744653.10^{* *}$ \\
\hline Testers & 4 & $0.50^{* *}$ & $119.76^{*}$ & $25.65^{* *}$ & $3.38^{* *}$ & $2614.74^{* *}$ & $777319.30^{* *}$ \\
\hline Lines $\boldsymbol{v s}$ Testers & 1 & 0.00061 & $1141.70^{* *}$ & $94.96^{*}$ & $1.66^{*}$ & $4188.18^{* *}$ & $161892.00^{* *}$ \\
\hline Parents vs Hybrids & 1 & $1.50^{* *}$ & $48053.65^{* *}$ & $265.24^{* *}$ & 0.37 & $593217.10^{* *}$ & $1428014.00^{* *}$ \\
\hline Hybrids & 29 & $0.27^{* *}$ & $1458.15^{* *}$ & $32.11^{* *}$ & 1.09 & $18964.78^{* *}$ & $366767.80^{* *}$ \\
\hline Error & 80 & 0.13 & 36.98 & 5.43 & 0.18 & 517.08 & 18290.00 \\
\hline
\end{tabular}

Fig.1 Pollens as captured after staining with 2\% aceto-carmine solution in CMS lines, Restorer lines, standard checks and $\mathrm{F}_{1}$ hybrids

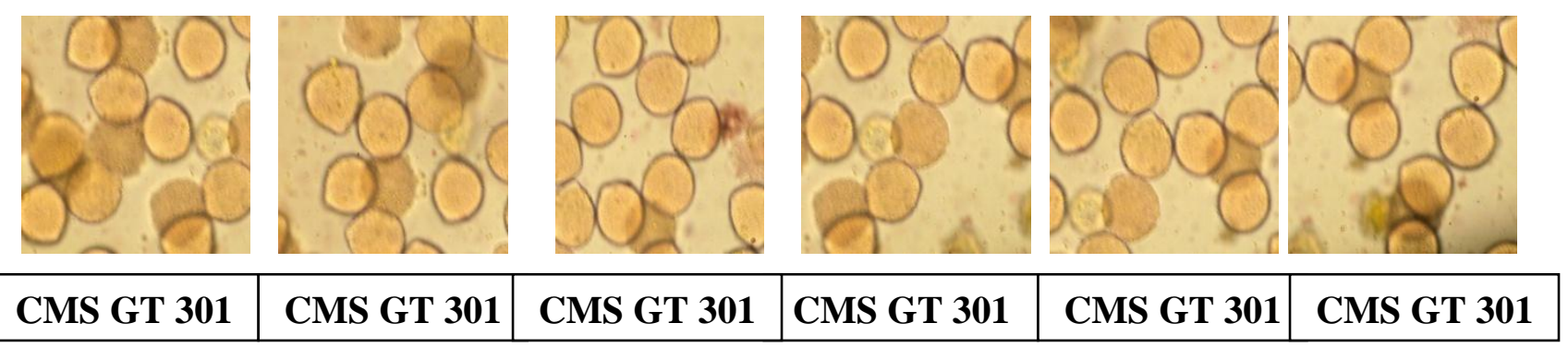

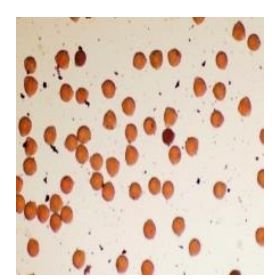

\section{GTR 8}
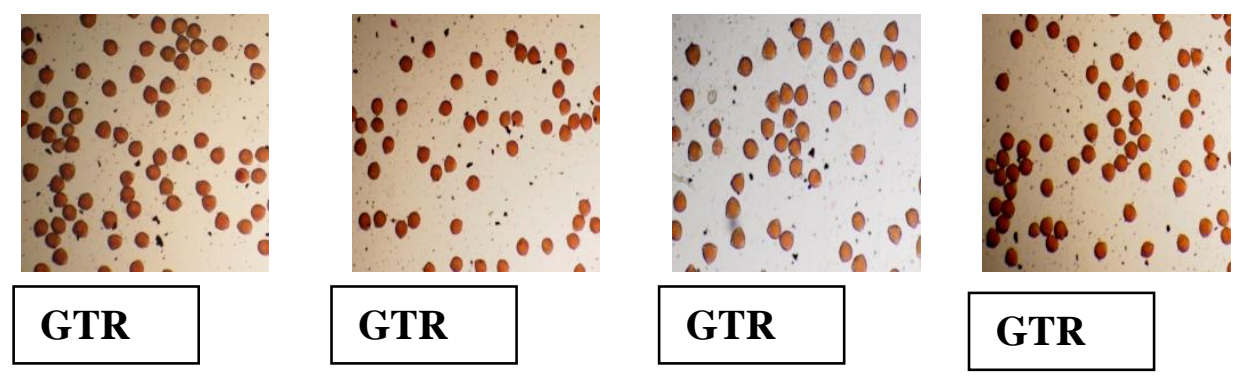

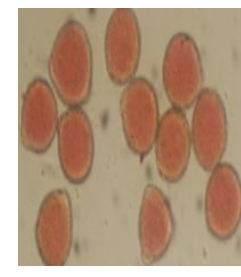

GTH 1

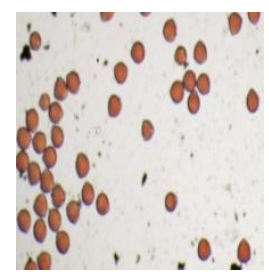

CMS GT 301 A X GTR 8

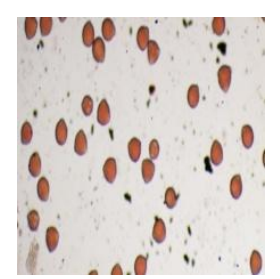

CMS GT 301 A X GTR 18

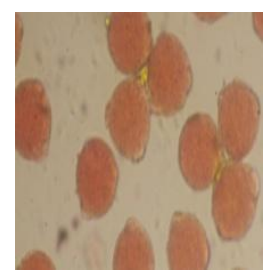

GT

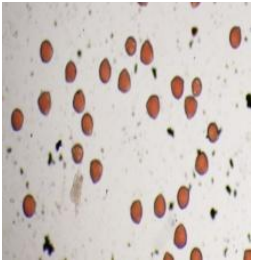

CMS GT 301 A X GTR 23

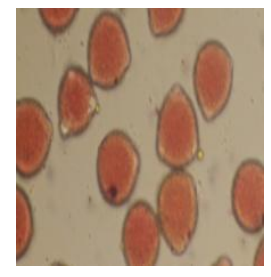

VAISH

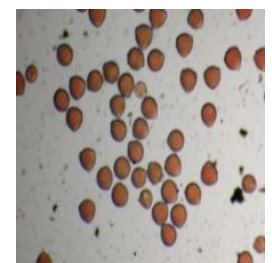
A X GTR 52

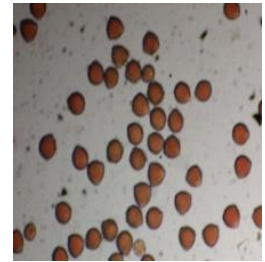

CMS GT 301 A X GTR 95 

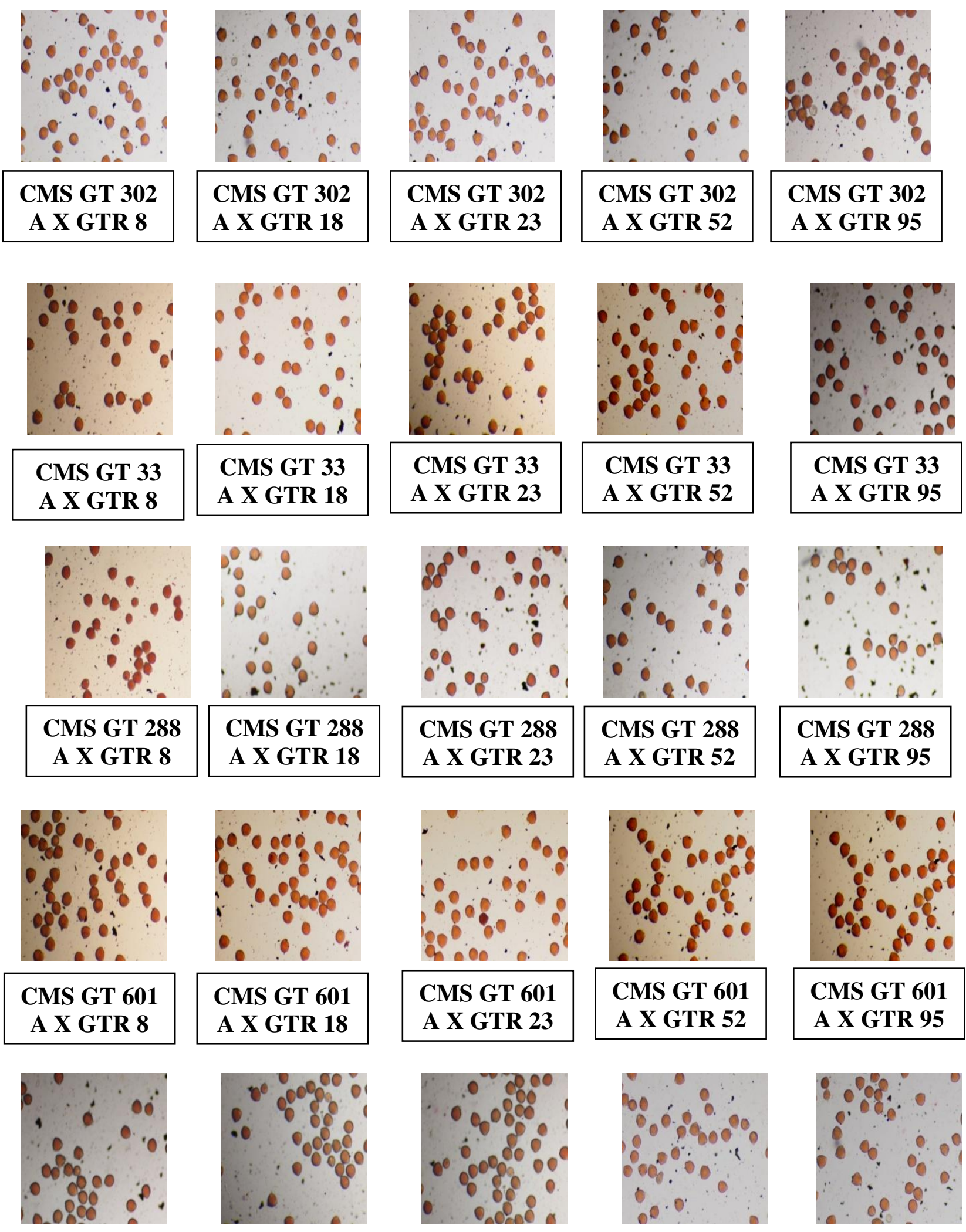

CMS GT 603 A X GTR 8
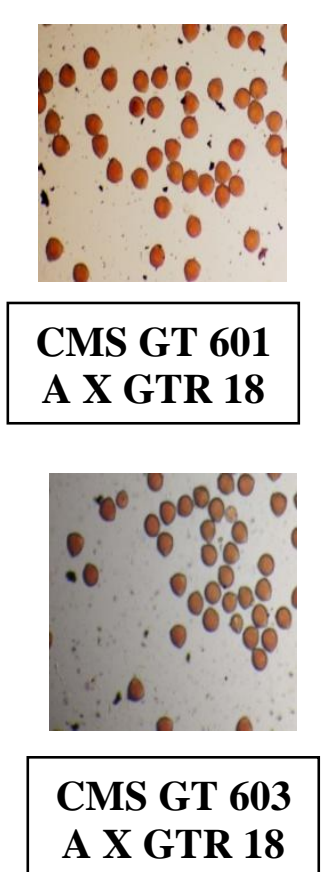
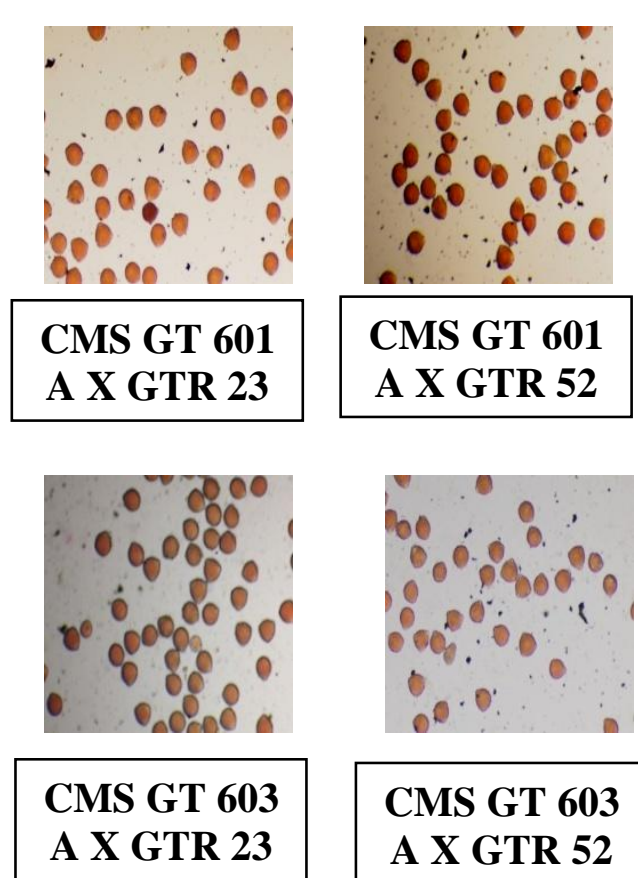

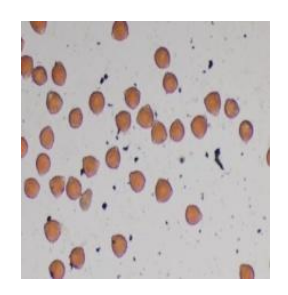

CMS GT 603 A X GTR 52
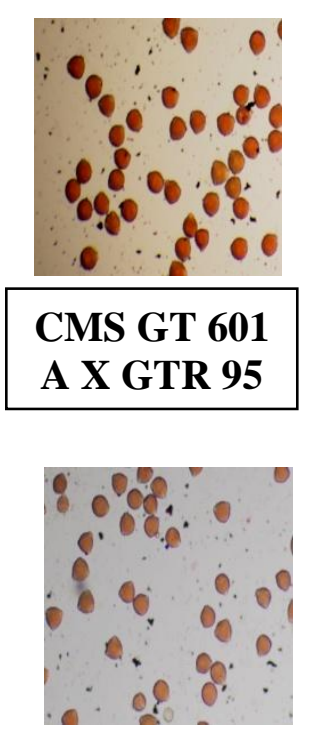

CMS GT 603 A X GTR 95 
Table.2 Estimates of standard heterosis (over GTH 1) for different traits

\begin{tabular}{|c|c|c|c|c|c|c|c|c|}
\hline Sr. & \multirow[t]{2}{*}{ Hybrids } & DF & DM & PH & BPP & $\mathbf{P P}$ & SPP & PL \\
\hline No & & SC & SC & SC & SC & SC & SC & $\mathrm{SC}$ \\
\hline 1. & CMS GT 301 A X GTR 8 & $20.42 * *$ & $14.84 * *$ & -0.68 & -11.92 & $-15.91 * *$ & $-22.52 * *$ & -3.10 \\
\hline 2. & CMS GT 301 A X GTR 18 & $22.18^{* *}$ & $18.19^{* * *}$ & 10.00 & -8.59 & -6.11 & $-24.21^{* *}$ & -6.64 \\
\hline 3. & CMS GT 301 A X GTR 23 & $20.42 * *$ & $14.60^{* * *}$ & 5.95 & $19.62 * *$ & $-11.42 *$ & $-14.53 * *$ & -5.97 \\
\hline 4. & CMS GT 301 A X GTR 52 & $20.07 * *$ & $16.27 * *$ & 6.88 & -13.72 & $-36.00 * *$ & 1.69 & -3.54 \\
\hline 5. & CMS GT 301 A X GTR 95 & $16.54 * *$ & $16.51^{* *}$ & $19.35^{\text {** }}$ & $27.31^{* *}$ & $15.97^{* * *}$ & -4.84 & -0.22 \\
\hline 6. & CMS GT 302 A X GTR 8 & $9.51 * *$ & $6.94 *$ & 6.88 & 10.26 & 0.12 & $-12.83^{* *}$ & -3.98 \\
\hline 7. & CMS GT 302 A X GTR 18 & $15.14 * *$ & $14.35^{* * *}$ & $13.77 *$ & $-13.72^{*}$ & $-13.98 * *$ & 1.69 & $16.15^{* *}$ \\
\hline 8. & CMS GT 302 A X GTR 23 & $12.68^{* *}$ & 3.83 & $13.40^{*}$ & $-16.28 * *$ & -8.49 & $-12.83^{* *}$ & 2.43 \\
\hline 9. & CMS GT 302 A X GTR 52 & $10.56^{* *}$ & $10.53^{* * *}$ & 11.76 & 0.00 & $-13.17 * *$ & 0.00 & $11.73^{*}$ \\
\hline 10. & CMS GT 302 A X GTR 95 & 11.97 ** & $11.01 * *$ & 10.70 & 9.36 & $-13.83 * *$ & -3.15 & $14.38 * *$ \\
\hline 11. & CMS GT 33 A X GTR 8 & $-10.21 * *$ & -1.44 & -2.89 & $-13.72^{*}$ & $-33.98 * *$ & $-15.98 * *$ & $10.84^{*}$ \\
\hline 12. & CMS GT 33 A X GTR 18 & $-4.58 *$ & 3.11 & 10.51 & $-21.41 * *$ & 4.33 & $-19.37^{* * *}$ & -2.43 \\
\hline 13. & CMS GT 33 A X GTR 23 & $-8.45^{* *}$ & -3.59 & 8.74 & 9.36 & $-14.32 * *$ & -3.15 & 2.21 \\
\hline 14. & CMS GT 33 A X GTR 52 & $-11.62 * *$ & -2.15 & -4.47 & $-14.49^{*}$ & $-24.05^{* *}$ & $-17.68^{* *}$ & $11.06^{*}$ \\
\hline 15. & CMS GT 33 A X GTR 95 & $-13.03 * *$ & -2.87 & 10.14 & $15.77^{*}$ & -6.42 & 1.69 & 2.21 \\
\hline 16. & CMS GT 288 A X GTR 8 & -1.42 & 3.59 & 3.77 & $-19.62 * *$ & $-11.19 *$ & 0.00 & $13.50^{* *}$ \\
\hline 17. & CMS GT 288 A X GTR 18 & 1.06 & $8.61 * *$ & 8.83 & -11.15 & -3.10 & $-17.68 * *$ & 6.86 \\
\hline 18. & CMS GT 288 A X GTR 23 & 0.00 & $9.09 * *$ & 0.37 & $17.95^{*}$ & $-27.50 * *$ & 1.69 & 9.07 \\
\hline 19. & CMS GT 288 A X GTR 52 & -0.36 & $8.38 * *$ & 10.88 & -11.92 & $-15.18 * *$ & 0.00 & $11.50^{*}$ \\
\hline 20. & CMS GT 288 A X GTR 95 & 0.00 & 3.35 & $19.26^{* *}$ & 1.67 & $12.38^{*}$ & -7.99 & $23.67 * *$ \\
\hline 21. & CMS GT 601 A X GTR 8 & $17.96^{* * *}$ & $13.64 * *$ & 5.72 & -10.26 & $-15.90 * *$ & -7.99 & $11.28^{*}$ \\
\hline 22. & CMS GT 601 A X GTR 18 & $17.96^{* *}$ & $13.88^{* * *}$ & 12.46 & $-15.38^{*}$ & -4.08 & -3.15 & 6.42 \\
\hline 23. & CMS GT 601 A X GTR 23 & $19.36^{* * *}$ & $18.90^{* * *}$ & 7.67 & -7.69 & $-21.64 * *$ & -7.99 & 3.54 \\
\hline 24. & CMS GT 601 A X GTR 52 & $14.79 * *$ & $13.40^{* * *}$ & 9.53 & $-21.41 * *$ & -0.91 & -3.15 & 7.30 \\
\hline 25. & CMS GT 601 A X GTR 95 & $19.71 * *$ & $14.12^{* * *}$ & $18.98 * *$ & -5.13 & $10.49 *$ & $-14.53 * *$ & 7.74 \\
\hline 26. & CMS GT 603 A X GTR 8 & 16.19 ** & $12.92 * *$ & $28.98^{* *}$ & -1.67 & -0.56 & $-14.53 * *$ & $10.40^{*}$ \\
\hline 27. & CMS GT 603 A X GTR 18 & 15.49 ** & $16.03^{* * *}$ & $21.53^{* *}$ & $-16.28^{*}$ & -6.88 & 1.69 & $15.93 * *$ \\
\hline 28. & CMS GT 603 A X GTR 23 & $19.36^{* * *}$ & $16.99 * *$ & $35.95^{* *}$ & $-13.72^{*}$ & $15.24 * *$ & 1.69 & $11.50^{*}$ \\
\hline 29. & CMS GT 603 A X GTR 52 & $14.08 * *$ & $14.12 * *$ & $41.76^{* * *}$ & 12.82 & $15.51 * *$ & $-12.83 * *$ & 6.19 \\
\hline 30. & CMS GT 603 A X GTR 95 & $16.19 * *$ & $13.40^{* * *}$ & $31.77 * *$ & $20.13 * *$ & $10.09 *$ & 1.69 & 10.18 \\
\hline & S.Em \pm & 2.054 & 3.20 & 9.478 & 0.5492 & 13.05 & 0.1585 & 0.2309 \\
\hline & $\begin{array}{l}\text { crosses showing significant } \\
\text { desirable heterosis }\end{array}$ & 5 & 0 & 0 & 5 & 6 & 0 & 12 \\
\hline
\end{tabular}




\begin{tabular}{|c|c|c|c|c|c|c|c|}
\hline \multirow{2}{*}{$\begin{array}{l}\text { Sr. } \\
\text { No. }\end{array}$} & \multirow{2}{*}{ Hybrids } & TSW & \multirow[t]{2}{*}{ SY } & HI & PC & \multirow[t]{2}{*}{ BY } & \multirow{2}{*}{$\begin{array}{l}\text { LA } \\
\text { SC }\end{array}$} \\
\hline & & SC & & SC & SC & & \\
\hline 1. & CMS GT 301 A X GTR 8 & 2.54 & -11.69 & -11.91 & $-7.68^{* *}$ & 0.92 & 0.92 \\
\hline 2. & CMS GT 301 A X GTR 18 & 3.74 & 9.55 & $-23.25 * *$ & 0.15 & 36.39 ** & $36.39 * *$ \\
\hline 3. & CMS GT 301 A X GTR 23 & -0.99 & -9.09 & $-23.68 * *$ & $-6.99 * *$ & $30.11^{* *}$ & $30.11 * *$ \\
\hline 4. & CMS GT 301 A X GTR 52 & -0.46 & $-27.29 * *$ & $-39.23 * *$ & $-4.11 *$ & $31.93 * *$ & $31.93 * *$ \\
\hline 5. & CMS GT 301 A X GTR 95 & 3.70 & $28.61 * *$ & $-30.32 * *$ & $-8.77 * *$ & 43.59 ** & $43.59 * *$ \\
\hline 6. & CMS GT 302 A X GTR 8 & $8.92 * *$ & $33.39 * *$ & 5.27 & $-4.46 * *$ & 29.30 ** & $29.30 * *$ \\
\hline 7. & CMS GT 302 A X GTR 18 & -0.67 & -3.45 & $-40.39 * *$ & $-9.02 * *$ & $40.44 * *$ & $40.44 * *$ \\
\hline 8. & CMS GT 302 A X GTR 23 & 4.51 & 3.48 & $-19.78 * *$ & $-7.83^{* *}$ & $38.46^{* *}$ & $38.46^{* *}$ \\
\hline 9. & CMS GT 302 A X GTR 52 & -0.07 & 7.81 & $-22.82 * *$ & $-8.67 * *$ & $33.36^{* *}$ & $33.36^{* *}$ \\
\hline 10. & CMS GT 302 A X GTR 95 & -0.35 & 3.91 & $-21.25 * *$ & $-6.24 * *$ & $36.11 * *$ & $36.11 * *$ \\
\hline 11. & CMS GT 33 A X GTR 8 & -4.02 & $-16.45 *$ & $-21.41 * *$ & 2.03 & -9.77 & -9.77 \\
\hline 12. & CMS GT 33 A X GTR 18 & 3.28 & $47.69 * *$ & 3.94 & 0.94 & $33.24 * *$ & $33.24 * *$ \\
\hline 13. & CMS GT 33 A X GTR 23 & -1.59 & -4.32 & $-18.45 * *$ & $-8.52 * *$ & $31.42 * *$ & $31.42 * *$ \\
\hline 14. & CMS GT 33 A X GTR 52 & $7.05^{*}$ & $-17.75 * *$ & $-21.35^{* *}$ & $-7.93 * *$ & -6.65 & -6.65 \\
\hline 15. & CMS GT 33 A X GTR 95 & 1.69 & $27.76^{* *}$ & -8.77 & $-6.84 * *$ & $32.83^{* *}$ & $32.83^{* *}$ \\
\hline 16. & CMS GT 288 A X GTR 8 & 0.00 & -3.02 & $-19.91 * *$ & $-7.43 * *$ & -4.41 & -4.41 \\
\hline 17. & CMS GT 288 A X GTR 18 & 2.50 & 8.68 & $-19.51 * *$ & $-6.24 * *$ & 29.60 ** & $29.60^{* * *}$ \\
\hline 18. & CMS GT 288 A X GTR 23 & -0.78 & $-21.65 * *$ & $-18.85 * *$ & $-8.82 * *$ & 4.32 & 4.32 \\
\hline 19. & CMS GT 288 A X GTR 52 & $8.43 * *$ & -3.02 & $-27.45^{* *}$ & $-7.58 * *$ & $35.19^{* *}$ & $35.19 * *$ \\
\hline 20. & CMS GT 288 A X GTR 95 & -1.52 & $57.22 * *$ & $-16.81 * *$ & $-7.04 * *$ & $39.79 * *$ & $39.79 * *$ \\
\hline 21. & CMS GT 601 A X GTR 8 & 5.01 & $-13.85^{*}$ & $-27.52 * *$ & $-8.57 * *$ & $28.02 * *$ & $28.02 * *$ \\
\hline 22. & CMS GT 601 A X GTR 18 & -0.07 & $15.18^{*}$ & $-21.65 * *$ & $-4.91 * *$ & $33.74 * *$ & $33.74 * *$ \\
\hline 23. & CMS GT 601 A X GTR 23 & -2.19 & $-24.25 * *$ & $-39.53 * *$ & $-3.87 *$ & $23.09^{* *}$ & $23.09 * *$ \\
\hline 24. & CMS GT 601 A X GTR 52 & -0.11 & $37.30^{* *}$ & 0.40 & $-4.06^{*}$ & 28.31 ** & $28.31 * *$ \\
\hline 25. & CMS GT 601 A X GTR 95 & 4.20 & $49.41 * *$ & -8.81 & $-9.12 * *$ & $44.95^{* *}$ & $44.95^{* *}$ \\
\hline 26. & CMS GT 603 A X GTR 8 & 4.44 & $46.81 * *$ & $-20.88 * *$ & $-8.33 * *$ & $56.38^{* *}$ & $56.38^{* *}$ \\
\hline 27. & CMS GT 603 A X GTR 18 & 0.35 & $21.68^{* *}$ & $-27.95 * *$ & $-7.93 * *$ & $49.04 * *$ & $49.04 * *$ \\
\hline 28. & CMS GT 603 A X GTR 23 & -0.95 & $64.59 * *$ & $-19.21 * *$ & $-9.56^{* *}$ & $57.74 * *$ & $57.74 * *$ \\
\hline 29. & CMS GT 603 A X GTR 52 & -0.21 & $69.79 * *$ & $-17.78 * *$ & $-7.93 * *$ & $62.93^{* *}$ & $62.93 * *$ \\
\hline 30. & CMS GT 603 A X GTR 95 & -0.49 & $53.76^{* *}$ & $-19.45 * *$ & $-7.53 * *$ & $55.21 * *$ & $55.21 * *$ \\
\hline \multirow{2}{*}{\multicolumn{2}{|c|}{$\begin{array}{c}\text { S.Em } \pm \\
\begin{array}{c}\text { No. of crosses showing significant } \\
\text { desirable heterosis }\end{array}\end{array}$}} & 0.3008 & 4.965 & 1.901 & 0.3533 & 18.56 & 110.42 \\
\hline & & 3 & 13 & 0 & 0 & 9 & 25 \\
\hline
\end{tabular}

Table.3 Best ten high yielding hybrids with heterosis (\%) over standard check (GTH 1) and showed high fertility restoration $(\%)$

\begin{tabular}{|c|c|c|c|c|c|}
\hline \multirow{2}{*}{$\begin{array}{l}\text { Sr. } \\
\text { No. }\end{array}$} & \multirow{2}{*}{$\begin{array}{l}\text { Ten best hybrids on the basis } \\
\text { of heterosis }(\%) \text { of seed yield }\end{array}$} & \multirow{2}{*}{$\begin{array}{l}\text { Mean seed } \\
\text { yield per } \\
\text { plant (g) }\end{array}$} & Heterosis (\%) & \multirow{2}{*}{$\begin{array}{c}\text { Significant standard } \\
\text { hetrosis for } \\
\text { component traits in } \\
\text { desired direction }\end{array}$} & \multirow{2}{*}{$\begin{array}{c}\text { Pollen Fertility } \\
\text { Restoration } \\
(\%)\end{array}$} \\
\hline & & & $\begin{array}{l}\text { SC } \\
\text { (GTH 1) }\end{array}$ & & \\
\hline 1. & CMS GT 603 A X GTR 52 & 130.60 & $69.79 * *$ & PP, BY, LA & 100.00 \\
\hline 2. & CMS GT 603 A X GTR 23 & 126.60 & $64.59 * *$ & PP, PL, BY, LA & 98.22 \\
\hline 3. & CMS GT 288 A X GTR 95 & 120.93 & $57.22 * *$ & PP, PL, BY, LA & 100.00 \\
\hline 4. & CMS GT 603 A X GTR 95 & 118.27 & $53.76^{* *}$ & PP, BY, LA, BPP & 94.42 \\
\hline 5. & CMS GT 601 A X GTR 95 & 114.93 & $49.41 * *$ & PP, BY, LA & 100.00 \\
\hline 6. & CMS GT 33 A X GTR 18 & 113.60 & $47.69 * *$ & LA, PC & 100.00 \\
\hline 7. & CMS GT 603 A X GTR 8 & 112.93 & $46.81 * *$ & LA, PL & 100.00 \\
\hline 8. & CMS GT 601 A X GTR 52 & 105.61 & $37.30 * *$ & LA & 95.302 \\
\hline 9. & CMS GT 302 A X GTR 8 & 102.60 & $33.39 * *$ & LA, TSW & 100.00 \\
\hline 10. & CMS GT 301 A X GTR 95 & 98.93 & $28.61 * *$ & PP, LA, BY, BPP & 94.33 \\
\hline
\end{tabular}


Table.4 Pollen fertility status recordrd for female, male parent, $F_{1}$ hybrids \& standard checks in pigeonpea

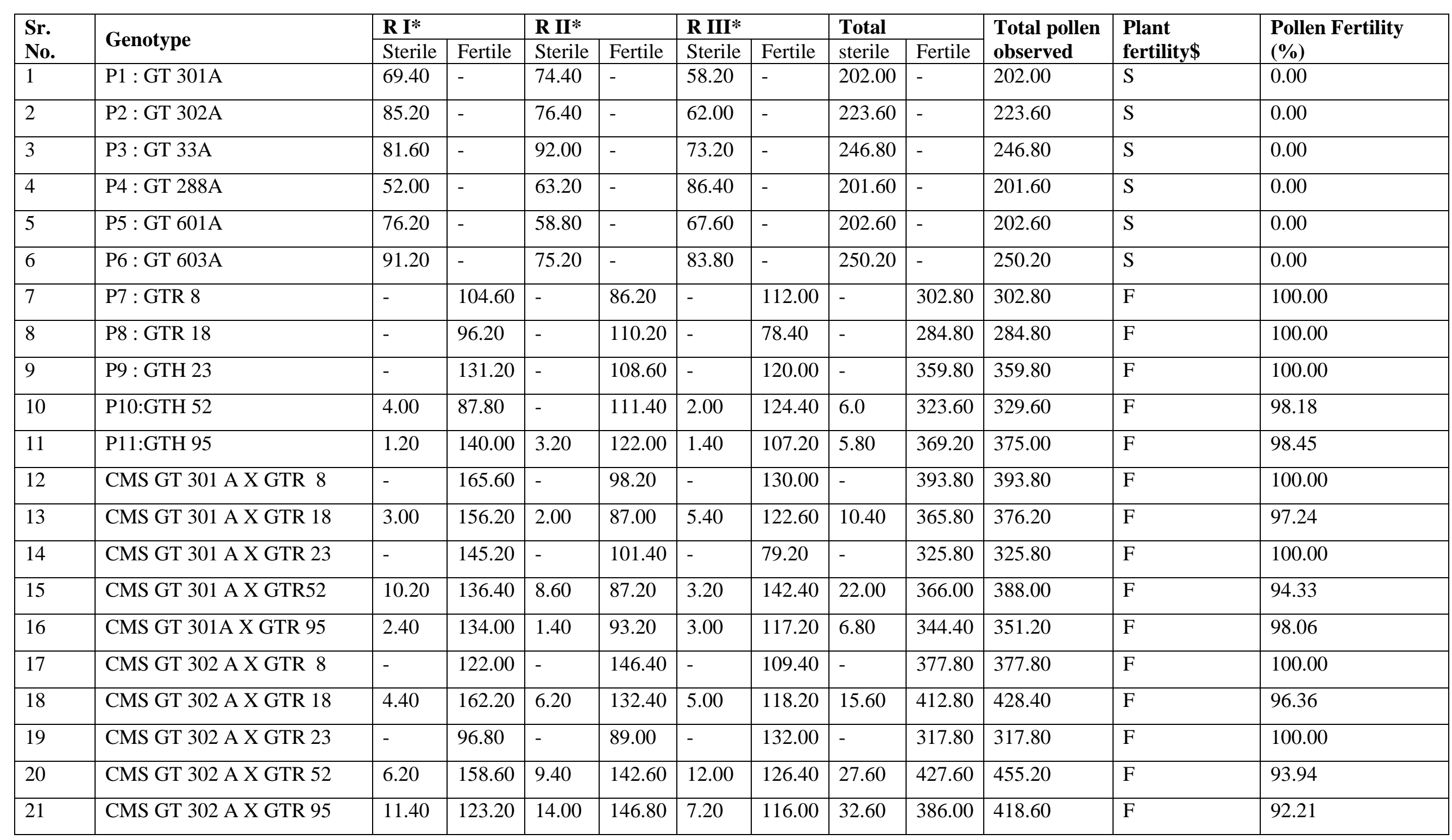




\begin{tabular}{|c|c|c|c|c|c|c|c|c|c|c|c|c|}
\hline 22 & CMS GT 33 A X GTR 8 & - & 98.20 & - & 133.20 & - & 153.20 & - & 384.60 & 384.60 & $\mathrm{~F}$ & 100.00 \\
\hline 23 & CMS GT 33 A X GTR 18 & - & 148.40 & - & 88.20 & - & 128.40 & - & 365.00 & 365.00 & $\mathrm{~F}$ & 100.00 \\
\hline 24 & CMS GT 33 A X GTR 23 & 3.60 & 137.00 & 1.40 & 130.00 & 3.40 & 143.00 & 8.40 & 410.00 & 418.40 & $\mathrm{~F}$ & 97.99 \\
\hline 26 & CMS GT 33 A X GTR 95 & - & 98.20 & - & 123.40 & - & 156.00 & - & 377.60 & 377.60 & $\mathrm{~F}$ & 100.00 \\
\hline 27 & CMS GT 288 A X GTR 8 & - & 167.60 & - & 128.20 & - & 107.20 & - & 403.00 & 403.00 & $\mathrm{~F}$ & 100.00 \\
\hline 28 & CMS GT 288 A X GTR 18 & 2.00 & 146.40 & 1.60 & 118.20 & 3.20 & 113.40 & 6.80 & 378.00 & 384.80 & $\mathrm{~F}$ & 98.23 \\
\hline 29 & CMS GT 288 A X GTR 23 & - & 127.00 & - & 138.60 & - & 106.20 & - & 371.80 & 371.80 & $\mathrm{~F}$ & 100.00 \\
\hline 30 & CMS GT 288 A X GTR 52 & 6.20 & 132.40 & 3.20 & 105.40 & 9.20 & 128.40 & 18.60 & 366.20 & 384.80 & $\mathrm{~F}$ & 95.17 \\
\hline 31 & CMS GT 288 A X GTR 95 & - & 112.20 & - & 153.00 & - & 147.80 & - & 413.00 & 413.00 & $\mathrm{~F}$ & 100.00 \\
\hline 32 & CMS GT 601 A X GTR 8 & 3.40 & 144.60 & 1.20 & 123.20 & 2.40 & 74.60 & 7.00 & 342.40 & 349.40 & $\mathrm{~F}$ & 98.00 \\
\hline 33 & CMS GT 601 A X GTR 18 & - & 163.00 & - & 116.40 & - & 135.00 & - & 414.40 & 414.40 & $\mathrm{~F}$ & 100.00 \\
\hline 34 & CMS GT 601 A X GTR 23 & 4.20 & 78.40 & 3.00 & 148.80 & 2.00 & 124.20 & 9.20 & 351.40 & 360.60 & $\mathrm{~F}$ & 97.45 \\
\hline 36 & CMS GT 601 A X GTR 95 & - & 138.20 & - & 115.00 & - & 130.00 & - & 383.20 & 383.20 & $\mathrm{~F}$ & 100.00 \\
\hline 37 & CMS GT 603 A X GTR 8 & - & 156.60 & - & 121.20 & - & 103.60 & - & 381.40 & 381.40 & $\mathrm{~F}$ & 100.00 \\
\hline 38 & CMS GT 603 A X GTR 18 & 2.40 & 149.60 & 1.20 & 137.20 & 5.60 & 125.00 & 9.20 & 411.80 & 421.00 & $\mathrm{~F}$ & 97.81 \\
\hline 39 & CMS GT 603 A X GTR 23 & 3.20 & 89.20 & 2.00 & 148.20 & 1.40 & 127.00 & 6.60 & 364.40 & 371.00 & $\mathrm{~F}$ & 98.22 \\
\hline 40 & CMS GT 603 A X GTR 52 & - & 107.60 & - & 105.00 & - & 135.20 & - & 347.80 & 347.80 & $\mathrm{~F}$ & 100.00 \\
\hline 41 & CMS GT 603 A X GTR 95 & 11.20 & 136.00 & 7.40 & 162.40 & 5.00 & 101.20 & 23.60 & 399.60 & 423.20 & $\mathrm{~F}$ & 94.42 \\
\hline 42 & GTH 1 & 6.40 & 96.40 & 1.00 & 103.80 & 2.20 & 115.60 & 9.60 & 315.80 & 325.40 & $\mathrm{~F}$ & 97.05 \\
\hline 43 & GT 101 & 3.00 & 121.40 & 2.60 & 89.60 & 1.80 & 106.40 & 7.40 & 317.40 & 324.80 & $\mathrm{~F}$ & 97.72 \\
\hline 44 & VAISHALI & 1.00 & 78.20 & 4.00 & 135.00 & 2.20 & 109.20 & 7.20 & 322.40 & 329.60 & $\mathrm{~F}$ & 97.82 \\
\hline
\end{tabular}

\footnotetext{
$*$ = Average of observations of five plants; $\$=$ Plant sterility: $\mathrm{S}=$ sterile and $\mathrm{F}=$ Fertile
} 
Fig.2 The graph showing pollen fertility status of different

CMS-based hybrids and check of pigeonpea

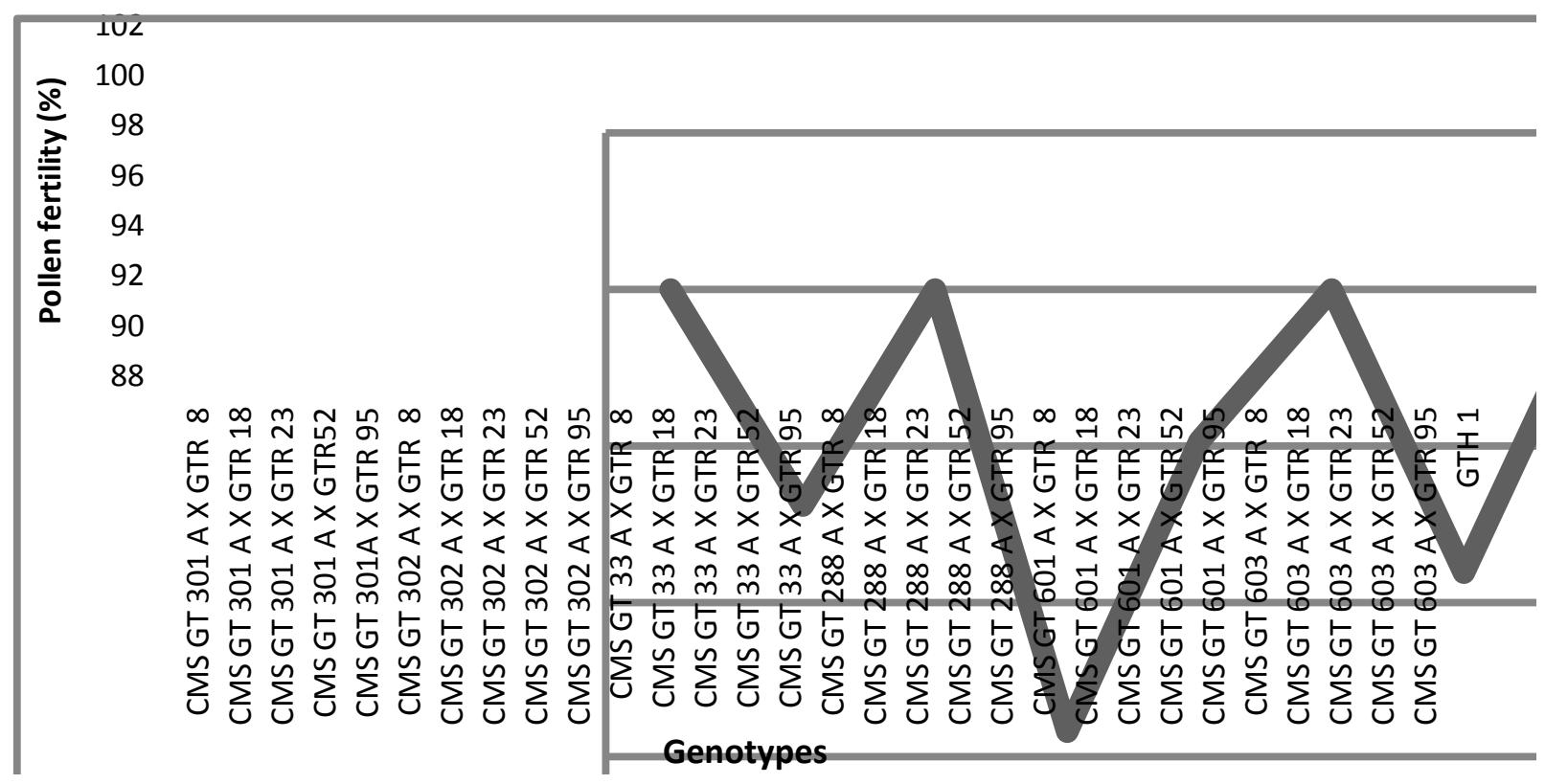

Harvest index indirectly influences the seed yield through partitioning photosynthates in source and sink. None of the hybrids reported (Table 2) significant and positive standard heterosis. It ranged from -40.39 (CMS GT 302 A X GTR 18) to 5.27 per cent (CMS GT 302 A X GTR 8). The results were in agreement with Patel et al., (1991), Aghav et al., (1997), Phad et al., (2009) and Gupta et al., (2011) for harvest index.

None of the hybrids exhibited significant positive heterosis against GTH 1 for protein content. The standard heterosis ranged from 9.56 (CMS GT 603 A X GTR 23) to 2.03 per cent (CMS GT 33 A X GTR 8). For biological yield per plant, nine hybrids exhibited significant and positive standard heterosis, that ranged from -34.12 (CMS GT 288 A X GTR 23) to 40.57 per cent (CMS GT 603 A X GTR 52) for GTH 1. These results were in close agreement with Baskaran and Muthiah (2006) and Patel and Tikka (2008) for protein content and biological yield per plant.
The growth of plant in term of mass will depend on how carbon is partitioned among new leaf area, leaf mass, root mass. The perusal of the results revealed (Table 2) that twenty five hybrids exhibited significant and positive standard heterosis. The range of standard heterosis was from -9.77 (CMS GT 33 A X GTR 8) to 62.93 per cent (CMS GT 603 A X GTR 52).

To confirm pollen fertility restoration in hybrids, the differences observed due to pollen fertility as tested pollen grains (with $2 \%$ acetocarmine solution) of parents (' $\mathrm{A}$ ' lines and male parents), hybrids and standard checks are given in Table 3 . The male sterile lines (P1) exhibited (100 per cent) pollen sterility. The pollen fertility in fertility restorer lines $(\mathrm{P} 2)$ varied from 98 to 100 per cent. The F1 generations of all the crosses exhibited pollen fertility restoration varying from 92 to 100 percent. Standard checks GTH 1, GTH 101 and VAISHALI exhibited 97, 98 and 97 per cent pollen fertility respectively. The pollen fertility was observed under light 
microscope and on the basis of its staining properties, pollen grains were categorized as fertile (deep to light red color) and sterile (shrivelled, hyaline, transparent) of different parents ( $\mathrm{P} 1$ and $\mathrm{P} 2)$, all the $\mathrm{F} 1$ crosses and standard checks given in Fig. 1 and data shown in FIG. 2 for fertility status of hybrids. The anthers of male sterile lines, restorer lines, F1 hybrids and standard checks were observed on visual basis for pollen fertility status, indicated male sterile lines had translucent type anthers whereas fertile lines (male parents, hybrids and checks) had normal pollen shedding in anthers. The results of present findings were in agreement to findings of Saxena and Kumar (2003), Chauhan et al., (2004), Mallikarjuna and Saxena (2005), Nadrajan et al., (2008), Kalaimagal et al., (2012), Saxena et al., (2013), Saxena et al., (2014) and Chaudhary et al., (2015).

From the study of heterosis and fertility restoration, it was concluded that to evaluate the restoration of fertility, Pollen fertility (\%) and amount of viable pollens produced by particular hybrid are an important characters which is a basic need for the successful production of high yielding CMS-based hybrids of pigeonpea. Due to success breeding of a stable CMS system and quality fertility restorers, we can standardized seed production technology.

The best cross combinations (Table 4) in order of merit seed yield and other yield components were CMS GT 603 A X GTR 52 (69.79\%), CMS GT 603 A X GTR 23 (64.59 $\%)$, CMS GT 288 A X GTR 95 (57.22\%) and CMS GT 603 A X GTR 95 (53.76 \%), CMS GT 601 A X GTR 95 (49.41 \%), CMS GT 33 A X GTR 18 (47.69 \%), CMS GT 603 A X GTR 8 (46.81\%), CMS GT 601 A X GTR 52 (37.30\%),CMS GT 302 A X GTR 8(33.39 $\%)$ and CMS GT 301 A X GTR 95 (28.61\%). These hybrids had high per se performance for grain yield, more standard heterosis with one or more its contributing traits viz. number of pods per plant, biological yield, leaf area and harvest index and having high pollen fertility status suggested that these hybrids can be directly exploited commercially after evaluating their performance in wide range of environment. These crosses can also be used to throw-off transgressive segregants in segregating generations for the improvement of yield and specific yield attributing traits.

\section{References}

Acharya, S., Patel, J.B., Patel, P.T. and Tikka, S.B.S. 2005. Characterization of stable and diversified CGMS (A) and restorer (R) lines of pigeonpea. Gujarat Agri. Univ. Res. J., 30(1-2): 1-7.

Acharya, S., Patel, J.B., Tank, C.J. and Yadav, A.S. 2009. Heterosis and combining ability Studies in Indo-African crosses of pigeonpea. J. Food Legumes, 22(2): 91-95.

Aghav, S.B., Narladkar, V.W. and Khapre, P.R. 1997. Heterosis for yield and yield components in pigeonpea hybrids. $J$. Maharashtra Agri. Univ., 22(1): 49-51.

Aher, G.U., Madrap, I.A., Tike, M.A. and Gore, D.R. 2006. Heterosis and inbreeding depression in pigeonpea. J. Maharashtra Agric. Univ., 31(1): 33-37.

Alexander, M.P. 1969. Differential staining of aborted and non-aborted pollen. Stain Technol., 44: 117-22.

Baskaran, K. and Muthiah, A.R. 2006. Interpretation of hybrid vigour in different cross combinations of pigeonpea [Cajanus cajan (L.) Millsp.]. Res. Crops, 7(1): 243248.

Chandirakala, R. and Raveendran, T.S. 2002. Heterosis in pigeonpea [Cajanus cajan (L.) Millsp.]. Annals Agri. Res., 23(2): 304-308.

Chandirakala, R., Subbaraman, N. and Hameed, A. 2010. Heterosis for yield in pigeonpea [Cajanus cajan (L.) Millsp.]. Electronic J. Plant Breeding, 1(2): 205-208.

Chaudhari, S., Tikle, A.N., Uttamchand, Saxena, K.B. and Rathore, A. 2015. Stability of cytoplasmic genetic male sterility and 
fertility restoration in pigeonpea. J. Crop Improvement, 29(3): 269-280

Chauhan, R.M., Parmar, L.D., Patel, P.T. and Tikka, S.B.S. 2004. Fertility restoration in cytoplasmic genetic male sterile line of pigeonpea [Cajanus cajan (L.) Millsp.] derived from Cajanus scarabaeoides. Indian J. Genetics Plant Breed., 64(2): 112114.

Dheva, N.G., Patil, A.N. and Wanjari, K.B. 2009. Heterosis in cytoplasmic male sterility based hybrids of pigeonpea. Int. J. Plant Sci., 4(1): 270-273.

FAOSTAT. 2006. FAOSTAT Agriculture data.

Gite, V.K., Madrap, I.A., Kamble, K.R. and Patil, D.K. 2009. Exploitation of heterosis in CMS based hybrid in pigeonpea, In: International Conference on Grain Legumes: Quality improvement, Value Addition and Trade, February, 2009. Indian Institute of Pulses Research, Kanpur, India. pp. 132-139.

Gupta, D.K., Acharya, S. and Patel, J.B. 2011. Combining ability and heterosis studies in pigeonpea using A2 cytoplasm from Cajanus scarabaeoides as source of male sterility. J. Food Legumes, 24(1): 58-64.

Kalaimagal, T., Muthiah, A.R. and Kumaresan, D. 2012. Inheritance of restoration for A2 cytoplasm of pigeonpea (Cajanus scarabaeoides). Crop Res., 43(1-3): 185188.

Kaul, M.L.H. 1988. Male sterility in higher plants. Springer Verlag Berlin, Heidelberg. New York 1005.

Kumar, A. and Srivastava, D.P. 1998. Heterosis in relation to combining ability in long duration pigeonpea. Indian J. Pulses Res., 11(2): 1-5.

Kumar, C.V.S., Sreelakshmi, C.H. and Varma, P.K. 2009. Studies on combining ability and heterosis in pigeon pea [Cajanus cajan (L.) Millsp.]. Legume Res., 32(2): 92-97.

Lay, K.K., Saxena, K.B., Kumar, R.V. and Rathore, A. 2011. Prospects of hybrids in enhancing production and productivity of pigeonpea in Myanmar. J. Food Legumes, 24(1): 1-7.

Majumder, N.D. 2004. All India Coordinated Research Project on Pigeonpea. Annual
Group Meet at MAU, Parbhani, 26-28 October.

Malikarjuna, N. and Saxena, K.B. 2005. A new cytoplasmic nuclear male-sterility system derived from cultivated pigeonpea cytoplasm. Euphytica, 142: 143-148.

Meredith, W.R. and Bridge, R.R. 1972. Heterosis and gene action in cotton ( $G$. hirsutum L.). Crop Sci., 12(3): 304-310.

Nadrajan, N., Ganesh, S.R. and Indira K.P. 2008. Fertility restoration studies in short duration redgram [Cajanus cajan (L.) Millspp.] Hybrids involving CGMS system. Madras Agri. J., 95(7-12): 320 - 327.

Patel, G.V., Zaveri, P.P. and Pathak, A.R. 1991. Heterosis for morphological attributes in pigeonpea. Indian J. Pulses Res., 4(1): 3541.

Patel, M.P. and Tikka, S.B.S. 2008. Heterosis for yield and yield components in pigeonpea. $J$. Food Legumes, 21(1): 65 - 66.

Patel, P.T., Chauhan, R.M., Parmar, L.D. and Tikka, S.B.S. 2004. Development of early maturing, high yielding pigeonpea hybrids for rainfed condition of Gujarat. In: National Symposium for arid legume for sustainable agriculture and trade, held on 5 to 7th November, 2004 at Jodhpur. pp. 26.

Patel, P.T. and Tikka, S.B.S. 2014a. Gene action and Stability parameters for yield and yield components, maturity duration and protein content of CGMS lines, pollen fertility restorers and their hybrids in pigeonpea [Cajanus cajan (L.) Millsp.]. Euphytica, 199: 349-362.

Patel, P.T. and Tikka, S.B.S. 2014b. Hybrid vigor in cytoplasmic geneic male sterility systembased hybrids for seed yield and its associated traits in pigeonpea [Cajanus cajan (L.) Millsp.]. Indian J. Genetics, 74(2): 257-260.

Phad, D.S., Madrap, I.A. and Dalvi, V.A. 2009. Heterosis in relation to combining ability effects and phenotypic stability in pigeonpea. J. Food Legumes, 22(1): 59-61.

Rana, S.G. 1990. Analysts of genetic variation, heterosis and maintenance of male sterile in pigeonpea [Cajanus cajan (L.) Millsp.]. M. Sc. Thesis (Unpublished) Gujarat Agricultural University, Sardarkrushinagar, Gujarat. 
Sarode, S.B., Singh, M.N. and Singh, U.P. 2009. Heterosis and combining ability analysis in long duration pigeonpea. In: International Conference on Grain Legumes: Quality Improvement, Value Addition and Trade, 14-16 February, 2009. Indian Institute of Pulses Research, Kanpur, India.

Saroj, S.K., Singh, M.N., Vishwakarma, M.K., Singh, T. and Mishra, V.K. 2015. Identification of stable restorers and genetics of fertility restoration in latematuring pigeonpea [Cajanus cajan (L.) Millspaugh]. J. Plant Breeding, 134(6): 696-702.

Savalia, D.V. 1994. Genetic studies in reation to hybrid pigeonpea [Cajanus cajan (L.) Millspaugh]. Ph.D. (Agri.) Thesis (Unpublished). Gujarat Agricultural University, Sardarkrushinagar.

Saxena, K.B. and Kumar, R.V. 2003. Development of a cytoplasmic-nuclear male sterility system in pigeonpea using $C$. scarabaeoides (L.) Thouars. Indian $J$. Genetics, 63: 225-229.

Saxena, K.B., Bharathi, M., Kumar, R.V., Srivastava, R.K. and Mallikarjuna, N. 2013. Studies on inheritance of male-sterility in Cajanus cajan and Cajanus acutifolius crosses. J. Food Legumes, 26(1-2): 26-29.

Saxena, K.B., Kumar, R.V. and Bharathi, M. 2014. Studies on fertility restoration of A4 cytoplasm in pigeonpea. Euphytica, 198: 127-135.

Saxena, K.B., Sultana, R., Saxena, R.K., Kumar, R.V., Sandhu, J.S., Rathore, A., Kishor, P.B.K. and Varshney, R.K. 2011. Fertility restoration in A4-based, diverse maturing hybrids of pigeonpea [Cajanus cajan (L.) Millsp.]. Crop Sci., 51(2): 574-587.

Shoba, D. and Balan, A. 2010a. Heterosis in CMS/GMS based pigeonpea [Cajanus cajan (L.) Millisp.] Hybrids. Agri. Sci. Digest, 30(1): 32-36.

Thiruvengadam, V. and Muthiaha, R. 2012. Combining ability analysis for yield and its components in pigeonpea using genetic male sterile lines. J. Food Legumes, 25(3): 171-174.

Tikka, S.B.S., Parmar, L.D. and Chauhan, R.M. 1997. First record of cytoplasmic genetic male sterility system in pigeonpea [Cajanus cajan (L.) Millsp.] Through wide hybridization. Gujarat Agri. Univ. Res. J., 22(2): 160-162.

Tiwari, P.N., Gambhi, P.N. and Rajan, T.S. 1974. Rapid and non-destructive determination of seed oil by pulsed Nuclear Magnetic Resonance Technique. J. Am. Oil Chemist's Soc., 51: 104-109.

Vaghela, K.O., Desai, R.T., Nizama, J.R., Patel, J.D. and Kodappully, V.C. 2011. Heterosis study for yield and yield components in pigeonpea. Res. Crops, 12(1): 192-194.

Wanjari, K.B., Bhongle, S.A. and Sable, N.H. 2007. Evaluation of heterosis in CMS based hybrids in pigeonpea. J. Food Legume, 20(1): 107-108.

Wankhade, R.R., Wanjari, K.B., Kadam, G.M. and Jadhav, B.P. 2003. Heterosis and combining ability in pigeonpea. In: National Symposium on Pulses for Crop Diversification and natural Resources management. Kanpur, 6: 60.

Wankhede, R.R., Wanjari, K.B., Kadam, G.M. and Jadhav, B.P. 2005. Heterosis for yield and yield components in pigeonpea involving male sterile lines. Indian $J$. Pulses Res., 18(2): 141-143.

Yadav, S.S. and Singh, D.P. 2004. Heterosis in pigeonpea. Indian J. Pulses Res., 17(2): 179-180.

\section{How to cite this article:}

Neetu Soni and Patel, P.T. 2017. Study of Heterosis and Pollen Fertility in Cgms Based Pigeonpea [Cajanus cajan (L.) Millspaugh] Hybrids. Int.J.Curr.Microbiol.App.Sci. 6(6): 956969. doi: https://doi.org/10.20546/ijcmas.2017.606.112 